4) W.D. Cornelius, J.M. Moss, and T. Yamaya, Phys. Rev. C 23, 1364 (1981); J.M. Moss, Phys. Rev. C 26, 727 (19 $\overline{82}$ ).

5) D.E. Bainum et al., Phys. Rev. Lett. 44, 1751 (1980); W.A. Sterrenburg, Sam M. Aust $\overline{1 n}$, R.P. DeVito, and Aaron Galonsky, Phys. Rev. Lett. 45, 1839 (1980); D.J. Horen et al., Phys. Lett. 998, 383 (1981); C. Gaarde et al., Nucl. Phys. A369, 258 (1981).
6) N. Auerbach, J.D. Bowman, M.A. Franey, and W.G. Love, Phys. Rev. C 28, 280, (1983); Phys. Rev. C 30, 736 (1984).

7) T. Izumoto, Nuc1. Phys - A395, 189 (1983); N. Auerbach and Amir Klein, Phys. Rev. C 30, 1032 (1984); F. Osterfeld, D. Cha, and J. Speth, Phys . Rev. C (to be published).

\title{
MEASUREMENTS OF GAMOW-TELLER STRENGTH DISTRIBUTIONS IN MASSES 13 AND 15
}

C.D. Goodman, R.C. Byrd, and I. van Heerden ${ }^{+}$

Indiana University Cyclotron Facility, Bloomington, Indiana 47405

\section{T.A. Carey}

Los Alamos National Laboratory, Los Alamos, New Mexico 87545

$$
\text { D.J. Horen }
$$

Oak Ridge National Laboratory, Oak Ridge, Tennessee 37830

\author{
J.S. Larsen, and C. Gaarde \\ Niels Bohr Institute, University of Copenhagen, DK-2100, Copenhagen, Denmark \\ J. Rapaport, and T.P. Welch \\ Ohio University, Athens, Ohio 45701 \\ E. Sugarbaker \\ Ohio State University, Columbus, Ohio 43214 \\ T.N. Taddeucci \\ Ohio University and Indiana University Cyclotron Facility
}

Allowed Fermi and Gamow-Teller beta decay

transition rates provide a special class of nuclear

model information because of the simple relationship

between the model description of the nucleus and the

transition process. The Fermi (F) operator changes

only the isospin projection of a nucleon. The

Gamow-Teller (GT) operator changes the projections of

both isospin and spin. The transition rate between

mirror states is the incoherent sum of the rates for

the Fermi and Gamow-Teller components. All of the

Fermi strength appears in the mirror state transition,

but due to the spin-orbit interaction, the GT strength

is distributed between the spin-orbit pair states, and

only a fraction of the total GT strength is contained

in the mirror state transition.
In mass 13 the ratio of measured ${ }^{1}$ and calculated ${ }^{2}$ GT strengths for the $1 / 2^{-} \rightarrow 1 / 2^{-}$mirror transition is 0.66. This ratio is consistent with the "typical" GT quenching factor. However, the calculated $B(G T)$ value for the strongest transition, 2 that from the ground state of ${ }^{13} \mathrm{C}$ to the $3 / 2^{-}, 3.51-\mathrm{MeV}$ level in ${ }^{13} \mathrm{~N}$, is $B(G T)=2.38$, while the value deduced from our $(p, n)$ measurements ${ }^{3}$ is $B(G T)=0.85 \pm 0.03$, a discrepancy of nearly a factor of three. The calculated B(GT) summed over all levels is 3.95 .

For mass 15, if we hold only to the restriction that the model space be limited to the p-shell, unlike the situation in mass 13, the total GT strength and the distribution of strength between the $p 1 / 2$ and $p 3 / 2$ hole states are independent of the spin-orbit splitting 
and of the two-body residual force. The total strength in this model is $B(G T)=3$, with $B(G T)=8 / 3$ going to the $p$ $3 / 2$ hole state and $B(G T)=1 / 3$ going to the $p 1 / 2$ hole state.

We have recently re-examined the distribution of strength in the mass 13 system and made new measurements in the mass 15 system. The normalization of our measurements requires that we know the fraction of the mirror state cross section attributable to GT strength. The previous method we used to deduce this fraction could be open to some question and we felt that it needed corroboration. We have now measured the spin-flip probability $S_{N N}\left(0^{\circ}\right)$ for $(p, n)$ reactions on ${ }^{13} \mathrm{C}$ and ${ }^{15} \mathrm{~N}$. This observable can be used to obtain a determination of the GT fractions independent of the method used in Ref. 3. In addition, we point out that both methods are independent of the absolute normalization of the $(p, n)$ cross sections and make use only of relative cross sections, which can be determined quite reliably.

The cross sections and transverse spin-flip probabilities for ${ }^{13} \mathrm{C}(\mathrm{p}, \mathrm{n})$ and ${ }^{15} \mathrm{~N}(\mathrm{p}, \mathrm{n})$ were measured at zero degrees using a $160 \mathrm{MeV}$ polarized proton beam from the Indiana University Cyclotron Facility and a neutron polarimeter consisting of bars of plastic scintillator at the end of a 45 meter flight path. The polarimeter is described briefly elsewhere 4 and will be described more fully in a future publication. The targets were pressed wafers of carbon $\left(>95 \%{ }^{13} \mathrm{C}\right)$ and Melamine $\left(\mathrm{C}_{3} \mathrm{H}_{6}{ }^{15} \mathrm{~N}_{6},>99 \%\right.$ enrichment in $\left.{ }^{15} \mathrm{~N}\right)$.

The results of our measurements are displayed in Table I, and the $(p, n)$ spectra are shown in Fig. 1 . The carbon contribution to the Melamine-target spectrum was subtracted by making use of data obtained under the same experimental conditions with a natural carbon target. Table I also shows calculated and

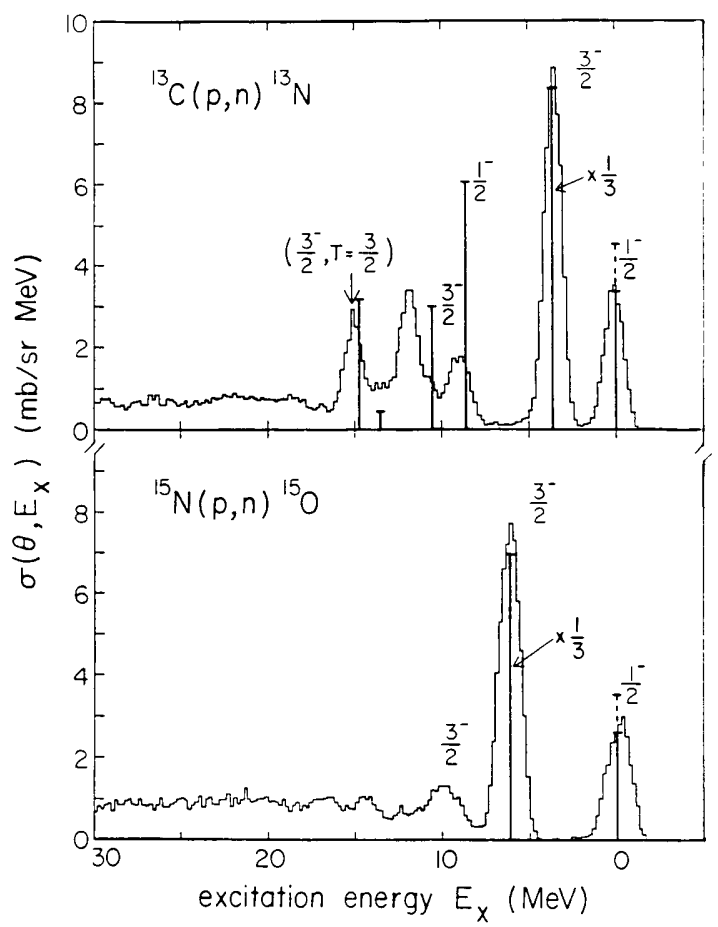

Figure 1. Spectra measured for the $(p, n)$ reaction on ${ }^{13} \mathrm{C}$ and $15 \mathrm{~N}$ at a proton energy of $160 \mathrm{MeV}$ and a scattering angle of $0^{\circ}$.

experimentally deduced values of $B(G T)$. The shell model calculations are based on the assumption that these nuclei can be characterized as p-shell nuclei. 2 The ground state to ground state $B(G T)$ values are deduced from beta decay ft values. We have used values frow Raman et al. 1

Excited-state transition strengths can be extracted by first decomposing the ground state cross section into Fermi and Gamow-Teller parts by using the observed relationship between GT and F transitions. 3

$\sigma_{G T} / \sigma_{F}=\left(E_{p} / 55 \pm 1 \mathrm{MeV}\right)^{2}[B(G T) / B(F)]$

where $B(F)=N-Z$. Once the fraction $f_{G T}=\sigma_{G T} /\left(\sigma_{G T}+\sigma_{F}\right)$ of the cross section attributable to the GT part of the mirror transition has been determined, the cross 
TABLE I. Cross sections, spin-flip probabilities, and GT transitions strengths for ${ }^{13} \mathrm{C}(p, n)$ and ${ }^{15} \mathrm{~N}(\mathrm{p}, \mathrm{n})$ at $\theta=0^{\circ}$ and $E_{\mathrm{p}}=160 \mathrm{MeV}$.

\begin{tabular}{lclll}
\hline Final State & $\begin{array}{c}\sigma\left(0^{\circ}\right)^{\mathrm{a}} \\
(\mathrm{mb} / \mathrm{sr})\end{array}$ & $\mathrm{S}_{\mathrm{NN}}\left(0^{\circ}\right)$ & $\mathrm{B}(\mathrm{GT})_{\text {expt }}$ & $B(G T)_{\text {model }}$ \\
\hline $13_{\mathrm{N}\left(0.0,1 / 2^{-}\right)}$ & $4.2 \pm 0.1$ & $0.46 \pm 0.02$ & $0.206 \pm 0.004^{\mathrm{b}}$ & $0.323^{\mathrm{e}}$ \\
$13_{\mathrm{N}\left(3.51,3 / 2^{-}\right)}$ & $10.5 \pm 0.1$ & $0.66 \pm 0.02$ & $0.83 \pm 0.03^{\mathrm{c}}$ & 2.38 \\
& & $0.75 \pm 0.05^{\mathrm{d}}$ & \\
\hline
\end{tabular}

\begin{tabular}{lclll}
\hline $150\left(0.0,1 / 2^{-}\right)$ & $4.5 \pm 0.1$ & $0.53 \pm 0.03$ & $0.261 \pm 0.006^{\mathrm{b}}$ & $1 / 3$ \\
$150\left(6.18,3 / 2^{-}\right)$ & $10.8 \pm 0.1$ & $0.70 \pm 0.03$ & $1.00 \pm 0.03^{\mathrm{c}}$ & $8 / 3$ \\
& & & $0.80 \pm 0.06^{\mathrm{d}}$ & \\
$150\left(8-12,3 / 2^{-}\right)$ & $3.2 \pm 0.1$ & $0.68 \pm 0.04$ & $0.30 \pm 0.02 \mathrm{c}$ & \\
& & & $0.26 \pm 0.02^{\mathrm{d}}$
\end{tabular}

a Statistical uncertainty only. Absolute normalization uncertainty is $\pm 15 \%$.

b Transition strength determined from $\beta^{+}$ft values, Ref. 1.

c $B(G T)$ determined from $(p, n)$ cross section ratios at $160 \mathrm{MeV}$ and the method of Eqs. (2) and (4). Note that the value for ${ }^{13} \mathrm{~N}(3.51 \mathrm{MeV})$ differs slightly from that in Ref. 4 because we have not averaged in values obtained at other energies.

d $B(G T)$ determined from $(p, n)$ data and Eqs. (3) and (4).

e Shell model transition strengths, Cohen-Kurath "POT" wave functions, Ref. 2 .

section per $B(G T)$ for that target is known. Values of $B(G T)$ for excited states are then extracted with this proportionality factor.

The spin-flip probability measurements give a second (independent) determination of the GT fraction in the ground state cross sections. The spin-flip probability for these transitions is the weighted sum of the pure $\mathrm{GT}$ value of $\mathrm{s}_{\mathrm{NN}}\left(0^{\circ}, \mathrm{GT}\right)=0.66 \pm 0.03$ and the Fermi value of zero. The "pure GT" value represents an average obtained from measurements of $S_{\text {NN }}$ for many GT transitions at $160 \mathrm{MeV} .4$ This value is consistent with the value $2 / 3$ expected for a pure $L=0$ transition. The uncertainty represents not only experimental uncertainties, but also real deviations from the nominal value that can be attributed to $L \neq 0$ amplitudes in the transition.

The two procedures for extracting the GT fraction in the ground state and $B(G T)$ for excited states can be summarized in the following formulas:

$$
\begin{aligned}
& \text { or } \mathrm{f}_{\mathrm{GT}}=\left[1+\mathrm{B}(\mathrm{F}) / \mathrm{B}_{\mathrm{M}}(\mathrm{GT}) \mathrm{R}^{2}\right]^{-1} \\
& \mathrm{f}_{\mathrm{GT}}=\mathrm{s}_{\mathrm{NN}}\left(0^{\circ}, \mathrm{M}\right) / \mathrm{s}_{\mathrm{NN}}\left(0^{\circ}, \mathrm{GT}\right) \\
& \text { and } \\
& \mathrm{B}_{\mathrm{X}}(\mathrm{GT})=\left(\sigma_{\mathrm{X}} / \sigma_{\mathrm{M}}\right) \mathrm{F}_{G T^{-1}} \mathrm{~B}_{\mathrm{M}}(\mathrm{GT}) F(q),
\end{aligned}
$$

where $R=E_{p} /(55 \pm 1 \mathrm{MeV}), M$ refers to the mirror (ground state) transition, and $x$ refers to the excited state. 
The factor $F(q)$ is a correction for the momentum transfer dependence of the differential cross sections and is constrained by $1.00<F(q)<1.10$ for the cases studied here. The values of $B(G T)$ for the excited states shown in Table I indicate that the methods of Eqs. (2) and (3) are more or less consistent. If anything, the spin-flip probability procedures makes the quenching of the $3 / 2^{-}$transitions look even greater.

For the strongest transitions in both mass 13 and mass 15 , the values of $B(G T)$ extracted by the above procedures are reduced from the lp shell-model values by factors much larger than typical GT quenching. A striking feature is that the model predicts a ratio of $8: 1$ for the $1 / 2 \rightarrow 3 / 2$ to $1 / 2 \rightarrow 1 / 2$ transitions in mass 15, yet the observed ratio is only about $4: 1$. Similarly, in mass 13 , a shell model calculation 2 predicts a ratio of $7.5: 1$ for the ratio of the strongest $1 / 2 \rightarrow 3 / 2$ transition to the mirror state $1 / 2 \rightarrow 1 / 2$ transition, while the observed value is about $4: 1$.

The $(p, n)$ data show for the first tine in clear isolation the comparison of GT transition strengths to different members of a spin-orbit pair. The data suggest that the $1 / 2 \rightarrow 3 / 2$ GT transitions are more quenched than the $1 / 2 \rightarrow 1 / 2$ transitions when compared to simple shell model calculations restricted to the $p$ she11. The nature of the model failure suggests that the model space for some nuclel generally considered to be p-shell must be enlarged beyond that shell and perhaps very much beyond that shell. The magnitude of the discrepancy indicates that some of the appealing simplicity of the nuclear shell model in providing a gulde to a valid truncation of the space seems to be 1ost. Even for one of the simplest shell model nuclei, the simple version of the model seems to fail.

Termanent address: University of the Western Cape, Bellville, South Africa.

1) S. Raman, C.A. Houser, T.A. Walkiewicz, and I.S. Towner, Atomic Data and Nuclear Data Tables 21, 567 (1978).

2) T.-S.H. Lee and D. Kurath, Phys. Rev. C 21, 293 (1980); D. Kurath, private communication, (1980); and S. Cohen and D. Kurath, Nucl. Phys. 73, 1 (1965).

3) T.N. Taddeucci, J. Rapaport, D.E. Bainum, C.D. Goodman, C.C. Foster, C. Gaarde, J. Larsen, C.A. Goulding, D.J. Horen, T. Masterson, and E. Sugarbaker, Phys. Rev. C 25, 1094 (1982); T.N. Taddeucci, in The Interaction Between Medium Energy Nucleons in Nuclei-1982, edited by H.0. Meyer, (AIP, New York, 1983), p. 228.

4) T.N. Taddeucc1, T.A. Carey, C. Gaarde, J. Larsen, C.D. Goodman, D.J. Horen, T. Masterson, J. Rapaport, T.P. Welch, and E. Sugarbaker, Phys. Rev. Lett. 52, 1960 (1984). 